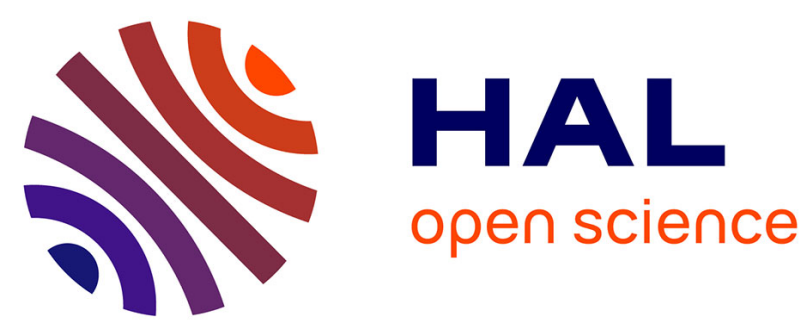

\title{
Effect of polynomial interpolations on the estimation performance of a frequency-selective Rayleigh channel in orthogonal frequency division multiplexing systems
}

Vincent Savaux, Moïse Djoko-Kouam, Yves Louët, Alexandre Skrzypczak

\section{- To cite this version:}

Vincent Savaux, Moïse Djoko-Kouam, Yves Louët, Alexandre Skrzypczak. Effect of polynomial interpolations on the estimation performance of a frequency-selective Rayleigh channel in orthogonal frequency division multiplexing systems. IET Signal Processing, 2015, 9 (1), pp.97-109. 10.1049/ietspr.2014.0053 . hal-01122211

\section{HAL Id: hal-01122211 \\ https://hal.science/hal-01122211}

Submitted on 3 Mar 2015

HAL is a multi-disciplinary open access archive for the deposit and dissemination of scientific research documents, whether they are published or not. The documents may come from teaching and research institutions in France or abroad, or from public or private research centers.
L'archive ouverte pluridisciplinaire HAL, est destinée au dépôt et à la diffusion de documents scientifiques de niveau recherche, publiés ou non, émanant des établissements d'enseignement et de recherche français ou étrangers, des laboratoires publics ou privés. 
This paper is a postprint of a

paper accepted by IET Signal Processing and is subject to Institution of Engineering and Technology Copyright. When the final version is published, the copy of record will be available at IET Digital Library 


\title{
Effect of Polynomial Interpolations on the Estimation Performance of a Frequency-Selective Rayleigh Channel in OFDM Systems
}

\author{
Vincent Savaux*, Moïse Djoko-Kouam, Yves Louët and Alexandre Skrzypczak
}

\begin{abstract}
In this article, we provide an analytical expression of the mean square error (MSE) and the bit error rate (BER) lower bound of an orthogonal frequency division multiplexing (OFDM) signal transmission over a multipath Rayleigh channel considering estimation errors. For some pilot arrangements, an interpolation is required to perform the channel estimation. Due to their low complexity, polynomial based interpolations are usually applied at the receiver, which induces estimation and signal errors. Based on a statistical analysis of these errors, the exact MSE expression of the channel estimation is provided. Furthermore, with a geometrical study of the constellation, an analytical BER limit is derived. For a given channel, it is shown that the errors are perfectly characterized by the interpolation method and the frequency gap between the pilot tones. All the steps of the analytical developments are validated through simulations. The proposed analysis then predicts the performance of the receiver, thus enabling the latter to a priori select the interpolation method with minimum complexity, according to a given channel and a BER target.
\end{abstract}

\section{Index Terms}

Bit error rate, Channel estimation, Interpolation, Mean square error, OFDM, Rayleigh channel.

Manuscript submitted 2014

* Vincent Savaux is with IETR-Supélec, Rennes, FR(corresponding author: e-mail: Vincent.Savaux@supelec.fr Phone: +33 299844536.)

Moïse Djoko-Kouam is with ECAM Rennes-Louis de Broglie, Rennes, FR (e-mail: moise.djoko-kouam@ecam-rennes.fr) Yves Louët is with IETR-Supélec, Rennes, FR (e-mail: Yves.Louet@supelec.fr)

Alexandre Skrzypczak is with Zodiac Data Systems, Caen, FR (e-mail: Alexandre.Skrzypczak@zodiacaerospace.com) 


\section{Effect of Polynomial Interpolations on the Estimation Performance of a Frequency-Selective Rayleigh Channel in OFDM Systems}

\section{INTRODUCTION}

Orthogonal frequency division multiplexing (OFDM) has become a very popular modulation scheme, for its robustness against the frequency selective channels. In order to estimate the channel frequency response, some pilots are inserted in the OFDM frame. A wide range of pilot-symbol-aided channel estimation techniques are described in [1]-[4]. Among them, and according to the pilot distribution, an interpolation may be necessary to estimate the channel over the time-frequency lattice. For instance, a comb-type pilot scheme or an arrangement in staggered row are adapted to the time and frequency selective channel, as in the digital radio mondiale (DRM/DRM+) [5] or the digital video broadcasting (DVB) [6] standard. These arrangements require an interpolation that may lead to estimation errors causing some decision errors and finally inducing a corrupted signal at the receiver. Consequently, the system performance, measured in bit error rate (BER) or mean square error (MSE) of estimation, reaches a lower limit even for a very low noise level.

The effects of the noise and the channel on the system performance have been studied for many years in the literature. In [7], the outcome of several channel models (e.g. Rayleigh, Rice, Nakagami) have been covered in order to express the theoretical symbol error rates (SER) of linear modulations in a single carrier context. The authors of [8] propose a closed-form of the exact BER expression for hierarchical modulations, and use the repetitive pattern of these kinds of constellations to derive a recursive algorithm for the exact BER computation [9]. In [10], the effects of the sinc channel estimation errors on the BER have been studied for a single carrier signal transmitted over a Rayleigh channel. This study has been extended in [11], in which the spatial diversity is considered at the receiver.

More recently, in an OFDM context, the authors of [12] study the lower bound reached by the BER curves due to different interpolation methods and for different constellation sizes. However, it does not provide any analytical expression of this BER lower bound. In [13], [14], an analytical expression of the SER and BER for a transmission in presence of channel frequency offset and channel estimation errors is derived for different modulation schemes. However, the developments are made by using the probability density functions (pdfs) given in [15], in which the channel coefficients and the error of estimation are supposed to be uncorrelated, which is not the case in practice.

In this article, we investigate the effect of interpolation methods based on low degrees polynomials, 
like the NN, linear or cubic interpolations [1], [2], and that do not need physical or statistical channel properties as for the linear minimum mean square error (LMMSE) [3], [4] or the maximum likelihood (ML) [16] techniques. As a matter of fact, these simple polynomial interpolation methods are the most used in practical receiver implementations, for they are much less complex than LMMSE or ML, although the latter are more accurate. Consequently, the analysis of their performance limits is of great interest. To this end, we characterize the system performance not only with a BER value as usually in the literature [10], [11], [13], [14], but also with the MSE of the channel estimation performed by means of the least square (LS) method with interpolations, as it is done in [4], [17] for the LMMSE. As in [10], [11], we use a Rayleigh channel model with correlated interpolation errors, but we consider the transmission of an OFDM signal over a frequency selective channel. To provide an analytical BER lower bound expression, we propose to study the statistics of the estimation errors and the geometry of the constellation. From the statistics of the errors, we then derive an analytical expression of the MSE for the LS estimation with interpolation. A general expression of the errors is provided, and is then applied to the nearest neighbor $(\mathrm{NN})$, the linear interpolations and the cubic interpolation methods.

The contributions of this article are the following ones: firstly, according to the channel parameters, we give an exact expression of the error statistics (variance, correlation coefficient, distribution) in function of the interpolation technique and the frequency gap between the pilot tones. Secondly, we propose an original approach for the exact BER floor calculation, by splitting the study into statistical and geometrical considerations. Thirdly, in addition to the BER analysis, the exact MSE of the LS estimation with interpolation is provided. This is an original performance study that highlights some interpolation properties, in particular regarding the interpolated noise. The proposed analysis can then be used at the receiver side to choose the less complex interpolation method for a BER target, and according to the channel.

The article is organized as follows: Section II describes the system model and gives the parameters used all along the paper. In Section III, the statistics of the errors are exactly expressed in function of the interpolation technique and the gap between the pilots. From these statistics, the mean square error of the estimations is analytically derived in Section IV, and then the exact BER floor value is given in Section V. The simulations results of Section VI validate the theoretical developments, and we draw conclusions and we give some prospects in Section VII.

\section{System Model}

\section{A. Background and Signal Model}

We consider the transmission of OFDM symbols over a multipath Rayleigh channel. After the removal of the cyclic prefix (CP) and the Discrete Fourier Transform (DFT) of size $M$, we express 
the received OFDM symbol vector in the frequency domain by

$$
\mathbf{U}=\underline{\mathbf{C H}}+\mathbf{W},
$$

where $\mathbf{U}=\left[U_{0}, \ldots, U_{M-1}\right]^{T}, \mathbf{H}=\left[H_{0}, \ldots, H_{M-1}\right]^{T}$ and $\mathbf{W}=\left[W_{0}, \ldots, W_{M-1}\right]^{T}$ denote the $M \times 1$ complex vectors of the received signal, the time and frequency selective channel and the Gaussian white noise of variance $\sigma^{2}$, respectively. The matrix $\underline{\mathbf{C}}$ is the $M \times M$ diagonal matrix of the transmitted signal containing the vector $\left[C_{0}, \ldots, C_{M-1}\right]$. Each $C_{m}$ is either a data element or a pilot, whose gain, phase and position are perfectly known at both transmitter and receiver sides. Since the channel is time-varying, let us assume OFDM symbols with a comb-type pilot arrangement [18], i.e. the pilot tones are evenly distributed in each symbol, with a frequency gap noted $\delta_{p}$. As reminded in [19], in order to capture all the channel frequency variations, this frequency gap must respect the Nyquist sampling theorem $\delta_{p} \leq 1 /\left(\tau_{\max } \Delta\left(f_{c}\right)\right)$, where $\tau_{\max }$ is the maximum delay spread and $\Delta\left(f_{c}\right)$ the subcarrier spacing. As illustrated in Fig. 1, three values $\delta_{p} \in\{2,3,4\}$ are considered, so as to generalize the further developments whatever $\delta_{p}$. For simulations purposes, each symbol has $M=241$ subcarriers. The reason is that $241=a \times \delta_{p}+1$, where $a$ is an integer. In order to only focus on the effect of the interpolation errors, the developments and the simulations are performed without considering channel coding.

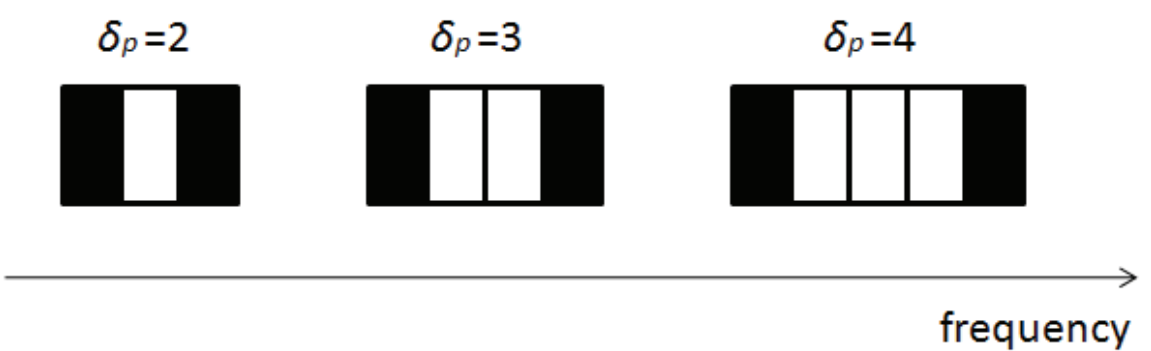

Fig. 1. Illustration of the pilot arrangements for different values of $\delta_{p}$, the pilot tones are black and the data carriers are white.

The frequency positions of the pilot tones are pointed out by the letter $p$, so as to write $f=f_{p}$ and $m=p$ in the continuous and in the discrete formalism, respectively. We also define $N_{p}$ the number of pilot tones per OFDM symbol. In the rest of the article and without loss of generality, the pilot are supposed to have a normalized power, that is $\left|C_{p}\right|^{2}=\mathcal{P}=1$.

\section{B. Channel Model}

Since polynomial-based interpolations methods are usually expressed for continuous functions, some developments will be made in a continuous formalism. However, it will be shown that the 
developments remain valid in the discrete formalism. Thus, the Rayleigh channel frequency response will be equivalently expressed by

$$
H(f)=\sum_{l=0}^{L-1} h_{l} e^{-2 j \pi f \tau_{l}} \Longleftrightarrow H_{m}=\sum_{l=0}^{L-1} h_{l} e^{-2 j \pi \frac{\beta_{l} m}{M}},
$$

where $\beta_{l}=\tau_{l} / \tau_{s}$ are the sampled path delays, $m=f M \tau_{s}$ the sampled frequency, with $\tau_{s}$ the sampling time, $L$ is the number of paths and $h_{l}$ are the zero-mean independent complex Gaussian path coefficients, according to the wide sense stationary uncorrelated scattering (WSSUS) model described in [20]. We also define $\sigma_{l}^{2}=E\left\{\left|h_{l}\right|^{2}\right\}$ the variance of the $l^{\text {th }}$ path.

\section{Channel Estimation}

According to the pilot positions, the channel estimation is expressed by

$$
\hat{H}(f)=\left\{\begin{array}{ll}
\hat{H}(f)^{L S}=H(f)+\frac{W(f)}{C(f)} & \text { if } f=f_{p} \\
\hat{H}(f)^{i n t}+\left(\frac{W(f)}{C(f)}\right)^{i n t} & \text { if } f \neq f_{p}
\end{array} \Longleftrightarrow \hat{H}_{m}=\left\{\begin{array}{ll}
\hat{H}_{m}^{L S}=H_{m}+\frac{W_{m}}{C_{m}} & \text { if } m=p \\
\hat{H}_{m}^{i n t}+\left(\frac{W_{m}}{C_{m}}\right)^{i n t} & \text { if } m \neq p
\end{array},\right.\right.
$$

in the continuous and discrete formalisms, where $\hat{H}(f)^{L S}$ is the usual least square estimation [4], [21], and the superscipt int denotes the interpolated values of the channel and the noise. Since the channel and the noise are uncorrelated, the interpolation of the sum of the channel and noise is the sum of the respective interpolations. Consequently, it is possible to study the statistics of the interpolation errors $H_{m}-\hat{H}_{m}^{i n t}$ independently of the interpolated noise $\left(\frac{W_{m}}{C_{m}}\right)^{i n t}$. The statistics of the noise and the channel estimation errors will be then used in order to derive the analytical expressions of the mean square error (MSE) and the bit error rate (BER) floor.

\section{STATISTICS OF THE INTERPOLATION ERRORS}

We propose to derive the analytical MSE and BER floor for three frequently used polynomial interpolations. To this end, the second-order statistics of the interpolation errors are required, in particular the variance of the error and the joint probability density function (pdf) of the channel and the error. In this section, we then statistically characterize the interpolation errors without taking into account the noise. We first give a general formulation of the statistical analysis, and we then apply it to the $\mathrm{NN}$, the linear and the piecewise cubic interpolation methods.

\section{A. General Formulation}

From (3), for a given frequency $f \neq f_{p}$ and without taking into account the noise, the error induced by the interpolation, noted $e_{h}$ is defined by 


$$
e_{h}=\hat{H}(f)^{i n t}-H\left(f_{p}\right)
$$

Let us also define $\xi=\left|e_{h}\right|$ and the random Rayleigh process $r=|H(f)|$. Since the error in (4) is function of the channel, it is assumed that it has the same distributions as this latter, i.e. $e_{h}$ is a complex Gaussian process and $\xi$ a Rayleigh process, whose variance noted $\sigma_{\xi}^{2}$ must be defined. This assumption will be verified afterward. From (4), we also deduce that the error depends on the frequency gap $f-f_{p}$. In the discrete formalism, we denote by $k$ the gap between a pilot $p$ a given position $m=p+k$, as illustrated on Fig. 2 .

$$
\delta_{p}=2
$$

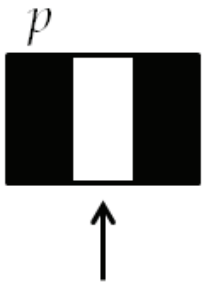

$\mathrm{k}=1$ $\delta_{p}=3$
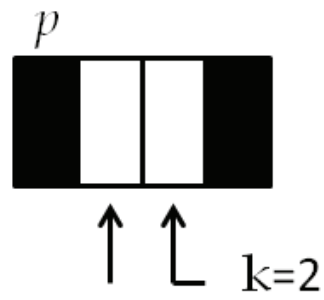

$\mathrm{k}=1$ $\delta_{p}=4$
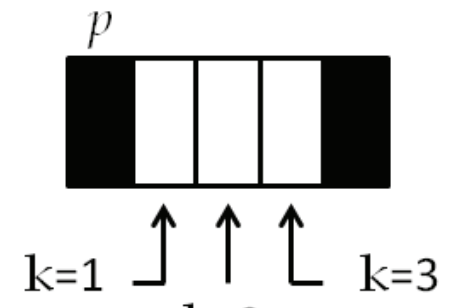

$\mathrm{k}=2$

Fig. 2. Discrete intervals for the nearest neighbor interpolation, for different values of $\delta_{p}$.

Thus, for a given position $m=p+k$ in the discrete interval $\rrbracket p, p+\delta_{p} \llbracket$, we define the error $\xi_{k}$ whose variance is denoted by $\sigma_{\xi_{k}}^{2}$. From Fig. 2, we observe that, by symmetry, $\xi_{k}$ has the same variance for $k=1$ and $k=2$ for $\delta_{p}=3$, and in the same way, $\sigma_{\xi_{1}}^{2}=\sigma_{\xi_{3}}^{2}$ for $\delta_{p}=4$. We deduce the probability density function of the error

$$
p_{\xi}(\xi)=\left\{\begin{array}{ll}
\frac{\xi}{\sigma_{\xi_{1}}^{2}} e^{-\frac{\xi^{2}}{2 \sigma_{\xi_{1}}^{2}}}, & \text { if } \delta_{p} \in\{2,3\} \\
\frac{2}{3}\left(\frac{\xi}{\sigma_{\xi_{1}}^{2}} e^{\left.-\frac{\xi^{2}}{2 \sigma_{\xi_{1}}^{2}}\right)+\frac{1}{3}\left(\frac{\xi}{\sigma_{\xi_{2}}^{2}} e^{-\frac{\xi^{2}}{2 \sigma_{\xi_{2}}^{2}}}\right),}\right. & \text { if } \delta_{p}=4
\end{array} .\right.
$$

The weights $2 / 3$ and $1 / 3$ in (5) correspond to the ratio between the symmetrical carriers having the same variances divided by the number of data-carriers in each discrete interval described in Fig. 2, for $\delta=4$. From (5), the general expression of $p_{\xi}(\xi)$ can be reasonably extrapolated (with an obvious recursion) whatever $\delta_{p}>2$ :

$$
p_{\xi}(\xi)=\left\{\begin{array}{ll}
\frac{1}{\delta_{p}-1} \frac{\xi}{\sigma_{\xi_{p} / 2}^{2}} e^{-\frac{\xi^{2}}{2 \sigma \xi_{\delta_{p} / 2}^{2}}}+\frac{2}{\delta_{p}-1} \sum_{k=1}^{\delta_{p} / 2-1} \frac{\xi}{\sigma_{\xi_{k}}^{2}} e^{-\frac{\xi^{2}}{2 \sigma_{\xi_{k}}^{2}}}, & \text { if } \delta_{p} \text { is even } \\
\frac{2}{\delta_{p}-1} \sum_{k=1}^{\left(\delta_{p}-1\right) / 2} \frac{\xi}{\sigma_{\xi_{k}}^{2}} e^{-\frac{\xi^{2}}{2 \sigma_{\xi_{k}}^{2}}}, & \text { if } \delta_{p} \text { is odd }
\end{array} .\right.
$$


The limit $\delta_{p} / 2-1$ and $\left(\delta_{p}-1\right) / 2$ in the sum are due to the fact that $\sigma_{\xi_{k}}^{2}=\sigma_{\xi_{\delta_{p}-k}}^{2}$.

It is obvious from (4) that the channel frequency response and the interpolation error are correlated. The model proposed in [15] is then not valid in this context. Since the channel $r$ and the error $\xi$ both follow a Rayleigh distribution, we know from [10], [22], [23] that the joint pdf of two correlated Rayleigh variables is

$$
p_{r, \xi}(r, \xi)=\frac{r \xi}{\sigma_{r}^{2} \sigma_{\xi}^{2}\left(1-\rho_{r \xi}^{2}\right)} \exp \left(-\frac{\sigma_{\xi}^{2} r^{2}+\sigma_{r}^{2} \xi^{2}}{2 \sigma_{r}^{2} \sigma_{\xi}^{2}\left(1-\rho_{r \xi}^{2}\right)}\right) I_{0}\left(\frac{r \xi \rho_{r \xi}}{\sigma_{r} \sigma_{\xi}\left(1-\rho_{r \xi}^{2}\right)}\right),
$$

where $\rho_{r \xi}$ is the correlation coefficient between the channel and the error of estimation due to the interpolation, and $I_{0}$ is the modified Bessel function of first kind with order zero. As for the variance $\sigma_{\xi}^{2}, \rho_{r \xi}$ depends on the interpolation method, and its expression will derived afterward. From (7), we also derive the conditional pdf $p_{\xi \mid r, \xi_{k}}\left(\xi \mid r, \xi_{k}\right)$ defined for a given value $k$ by $p_{\xi \mid r, \xi_{k}}\left(\xi \mid r, \xi_{k}\right)=$ $\frac{p_{r, \xi_{k}}\left(r, \xi_{k}\right)}{p_{r}(r)}$. Similarly as $\xi$, the generalized expression of the conditional pdf is also a weighted sum of the conditional pdfs for different $k$ values:

$$
p_{\xi \mid r}(\xi \mid r)=\left\{\begin{array}{ll}
\frac{1}{\delta_{p}-1} p_{\xi \mid r, \xi_{\delta_{p} / 2}}\left(\xi \mid r, \xi_{\delta_{p} / 2}\right)+\frac{2}{\delta_{p}-1} \sum_{k=1}^{\delta_{p} / 2-1} p_{\xi \mid r, \xi_{k}}\left(\xi \mid r, \xi_{k}\right), & \text { if } \delta_{p} \text { is even } \\
\frac{2}{\delta_{p}-1} \sum_{k=1}^{\left(\delta_{p}-1\right) / 2} p_{\xi \mid r, \xi_{k}}\left(\xi \mid r, \xi_{k}\right), & \text { if } \delta_{p} \text { is odd }
\end{array} .\right.
$$

This general statistics of the estimation errors are applied in the following, to the NN, the linear and the piecewise cubic interpolations.

\section{B. Nearest Neighbor Interpolation}

1) Reminder: Let us consider three consecutive frequency pilot positions $f_{p-\delta_{p}}, f_{p}$ and $f_{p+\delta_{p}}$, such as $f_{p}$ is not at the edge of the channel, i.e. $p \notin\{0, M-1\}$. From that, we denote $f_{p^{-}}$and $f_{p^{+}}$the centers of the intervals $\left[f_{p-\delta_{p}}, f_{p}\right]$ and $\left[f_{p}, f_{p+\delta_{p}}\right]$ respectively. $\forall f \in\left[f_{p^{-}}, f_{p^{+}}\right]$, the channel estimation made by a NN interpolation within this interval is simply written

$$
\hat{H}(f)=\hat{H}\left(f_{p}\right)^{L S}=H\left(f_{p}\right)+\frac{W\left(f_{p}\right)}{C\left(f_{p}\right)},
$$

where $\hat{H}\left(f_{p}\right)^{L S}$ is the LS estimation performed on the position $f_{p}$. If $p=0$ or $p=M-1$, the NN interpolation is limited to the intervals $\left[f_{0}, f_{0^{+}}\right]$and $\left[f_{M-1^{-}}, f_{M-1}\right]$, respectively, but the expression (9) remains the same.

2) Variance of the Errors for the NN Interpolation: By replacing $H$ by its value given by (2), it yields

$$
\xi_{N N}=\left|\sum_{l=0}^{L-1} h_{l} e^{-2 j \pi f_{p} \tau_{l}}\left(1-e^{-2 j \pi \Delta_{f} \tau_{l}}\right)\right|,
$$


where $\Delta_{f}=f-f_{p}$. As $e^{-2 j \pi f_{p} \tau_{l}}\left(1-e^{-2 j \pi \Delta_{f} \tau_{l}}\right)$ is deterministic, we conclude that, since $|H(f)|$ follows a Rayleigh distribution, then $\xi_{N N}$ has the same distribution. From (10), whatever $p=$ $0, \delta_{p}, . ., M-1$ and whatever $\delta_{p}$, we now express $\xi_{k, N N}$ the discrete version of $\xi_{N N}$ in (10):

$$
\xi_{k, N N}=\left|\sum_{l=0}^{L-1} h_{l} e^{-2 j \pi \frac{p \beta_{l}}{M}}\left(1-e^{-2 j \pi \frac{k \beta_{l}}{M}}\right)\right| .
$$

The variance of $\xi_{k, N N}$, noted $\sigma_{\xi_{k, N N}}^{2}$, is expressed

$$
\begin{aligned}
\sigma_{\xi_{k, N N}}^{2} & =E\left\{e_{h, N N} e_{h, N N}^{*}\right\} \\
& =E\left\{\left(\sum_{l_{1}=0}^{L-1} h_{l_{1}} e^{-2 j \pi \frac{p \beta_{l_{1}}}{M}}\left(1-e^{-2 j \pi \frac{k \beta_{l_{1}}}{M}}\right)\right)\left(\sum_{l_{2}=0}^{L-1} h_{l_{2}} e^{-2 j \pi \frac{p \beta_{l_{2}}}{M}}\left(1-e^{-2 j \pi \frac{k \beta_{l_{2}}}{M}}\right)\right)^{*}\right\} \\
& =E\left\{\sum_{l=0}^{L-1}\left|h_{l}\right|^{2}\left(2-e^{-2 j \pi \frac{k \beta_{l}}{M}}-e^{2 j \pi \frac{k \beta_{l}}{M}}\right)\right\} \\
& =\sum_{l=0}^{L-1} \sigma_{l}^{2}\left(2-2 \cos \left(2 \pi \frac{k \beta_{l}}{M}\right)\right) .
\end{aligned}
$$

We observe in (12) that, since $\sigma_{\xi_{k, N N}}^{2}$ depends on $k$, it consequently depends on the considered gap $\delta_{p}$. Thus, for $\delta_{p} \in\{2,3\},|k|=1$, so $\sigma_{\xi_{k, N N}}^{2}$ takes a sole value. For $\delta_{p}=4,|k|=1$ for two thirds of the estimated carriers in a discrete interval described in Fig. 2, and $|k|=2$ for one third. Thus, we deduce the variance $\sigma_{\xi_{N N}}^{2}$ of the error $\xi_{N N}$ (considered for all $k$ ):

$$
\sigma_{\xi_{N N}}^{2}= \begin{cases}\sigma_{\xi_{1, N N}}^{2}=\sum_{l=0}^{L-1} \sigma_{l}^{2}\left(2-2 \cos \left(2 \pi \frac{\beta_{l}}{M}\right)\right), & \text { if } \delta_{p} \in\{2,3\} \\ \frac{2}{3} \sigma_{\xi_{1, N N}}^{2}+\frac{1}{3} \sigma_{\xi_{2, N N}}^{2} & \\ =\frac{2}{3} \sum_{l=0}^{L-1} \sigma_{l}^{2}\left(2-2 \cos \left(2 \pi \frac{\beta_{l}}{M}\right)\right)+\frac{1}{3} \sum_{l=0}^{L-1} \sigma_{l}^{2}\left(2-2 \cos \left(2 \pi \frac{2 \beta_{l}}{M}\right)\right), & \text { if } \delta_{p}=4,\end{cases}
$$

3) Correlation coefficient $\rho_{r \xi}$ for the $N N$ Interpolation: The correlation coefficient, noted $\rho_{r \xi, N N}$ in the case of a $\mathrm{NN}$ interpolation, is expressed by

$$
\begin{aligned}
\rho_{r \xi, N N} & =\frac{\left|E\left\{H(f) e_{h}^{*}\right\}\right|}{\sigma_{h} \sigma_{\xi_{N N}}} \\
& =\frac{\left|\sum_{l=0}^{L-1} E\left\{\left|h_{l}\right|^{2}\left(e^{2 j \pi \tau_{l}\left(f_{p}-f\right)}-1\right)\right\}\right|}{\sigma_{h} \sigma_{\xi_{N N}}} \\
& =\frac{\left|\sum_{l=0}^{L-1} \sigma_{l}^{2}\left(e^{-2 j \pi \tau_{l} \Delta_{f}}-1\right)\right|}{\sigma_{h} \sigma_{\xi_{N N}}},
\end{aligned}
$$

with $\sigma_{h}$ the variance of the channel. In the discrete domain, the error depends on the value of $k$, so we obtain: 


$$
\rho_{r \xi_{k}, N N}=\frac{\left|\sum_{l=0}^{L-1} \sigma_{l}^{2}\left(e^{-2 j \pi \frac{\beta_{l} k}{M}}-1\right)\right|}{\sigma_{h} \sigma_{\xi_{k, N N}}} .
$$

\section{Linear Interpolation}

1) Reminder: In this section, as previously, a similar development is performed for the linear interpolation. We first remind the expression of the linear interpolation, for $f \in\left[f_{p}, f_{p+\delta_{p}}\right], \hat{H}(f)$ is obtained by

$$
\hat{H}(f)=\hat{H}^{L S}\left(f_{p}\right)+\left(f-f_{p}\right) \frac{\hat{H}^{L S}\left(f_{p+\delta_{p}}\right)-\hat{H}^{L S}\left(f_{p}\right)}{f_{p+\delta_{p}}-f_{p}},
$$

where $\hat{H}^{L S}\left(f_{p}\right)$ is the LS estimation performed on the $p^{t h}$ pilot tone. We remind that, in this section, we are only interested in the interpolation errors, so (16) is rewritten without the noise component:

$$
\hat{H}(f)^{i n t}=H\left(f_{p}\right)+\left(f-f_{p}\right) \frac{H\left(f_{p+\delta_{p}}\right)-H\left(f_{p}\right)}{f_{p+\delta_{p}}-f_{p}} .
$$

2) Variance of the Errors for the Linear Interpolation: For the linear interpolation, it is well known that the error between a given function $g$ of class $\mathcal{C}^{2}$ and its approximation $\hat{g}$ by a linear function at a point $x$ is given by

$$
|g(x)-\hat{g}(x)|=\frac{1}{2}\left|\left(x_{p+\delta_{p}}-x\right)\left(x_{p}-x\right)\right| \times\left|g^{\prime \prime}(y)\right|,
$$

where $x_{p}$ and $x_{p+\delta_{p}}$ are two consecutive nodes such as $\hat{g}\left(x_{p}\right)=g\left(x_{p}\right)$ and $\hat{g}\left(x_{p+\delta_{p}}\right)=g\left(x_{p+\delta_{p}}\right)$, and $y \in\left[x_{p}, x_{p+\delta_{p}}\right]$. This expression comes from the Taylor's expansion of $g(x)$ on $x_{p}$ and $x_{p+\delta_{p}}$. It is obvious that the channel frequency response (2) is $\mathcal{C}^{2}$ on $\left[f_{0}, f_{M-1}\right]$, so (18) can be used to determine the error of interpolation noted $\xi_{l i}$, on each interval $\left[f_{p}, f_{p+\delta_{p}}\right]$ :

$$
\xi_{l i}=|H(f)-\hat{H}(f)|=\frac{1}{2}\left|\left(f_{p+\delta_{p}}-f\right)\left(f_{p}-f\right)\right| \times\left|H^{\prime \prime}(\alpha)\right|,
$$

where $\alpha \in\left[f_{p}, f_{p+\delta_{p}}\right]$ and

$$
H^{\prime \prime}(f)=-4 \pi^{2} \sum_{l=1}^{L-1} h_{l} \tau_{l}^{2} e^{-2 j \pi f \tau_{l}} .
$$

It is straightforward from (20) that if $H(f)$ has a Rayleigh distribution, then $H^{\prime \prime}(f)$ follows a Rayleigh distribution as well. For further results, we deduce that, whatever $n \in \mathbb{N}$, the $n^{\text {th }}$ derivative $H^{(n)}(f)$ follows a Rayleigh distribution. We then deduce the variance of $H^{\prime \prime}(f)$ : 


$$
\begin{aligned}
\sigma_{H^{\prime \prime}}^{2} & =E\left\{H^{\prime \prime}(f) H^{\prime \prime}(f)^{*}\right\} \\
& =\sum_{l=1}^{L-1} E\left\{\left|4 \pi^{2} h_{l} \tau_{l}^{2}\right|^{2}\right\} \\
& =16 \pi^{4} \sum_{l=1}^{L-1} \tau_{l}^{4} \sigma_{l}^{2},
\end{aligned}
$$

and by inserting (21) into (19), we deduce the variance of $\xi_{l i}$ :

$$
\begin{aligned}
\sigma_{\xi_{l i}}^{2} & =\frac{1}{4}\left|\left(f_{p+\delta_{p}}-f\right)\left(f_{p}-f\right)\right|^{2} \sigma_{H^{\prime \prime}}^{2} \\
& =\left|\left(f_{p+\delta_{p}}-f\right)\left(f_{p}-f\right)\right|^{2} 4 \pi^{4} \sum_{l=1}^{L-1} \tau_{l}^{4} \sigma_{l}^{2} .
\end{aligned}
$$

In the discrete formalism, $\sigma_{\xi_{l i}}^{2}$ depends on the value $k$, and we note $\sigma_{\xi_{k, l i}}^{2}$ the variance given by

$$
\sigma_{\xi_{k, l i}}^{2}=\left|\frac{\left(\delta_{p}-k\right) k}{M^{2}}\right|^{2} 4 \pi^{4} \sum_{l=1}^{L-1} \beta_{l}^{4} \sigma_{l}^{2},
$$

for $k=1, . ., \delta_{p}-1$. One can notice if the channel tends to have flat fading (i.e. $\beta_{l}$ tends to zero), then $\sigma_{\xi_{k, l i}}^{2}$ logically tends to zero. Indeed, the linear interpolation of a constant function leads to an error equal to zero. The pdf of the error of the linear interpolation is denoted by $p_{\xi_{l i}}(\xi)$. It has exactly the same formulation than the $\mathrm{NN}$ interpolation given in (5), by replacing $\sigma_{\xi_{k, N N}}^{2}$ by $\sigma_{\xi_{k, l i}}^{2}$.

3) Correlation coefficient $\rho_{r \xi}$ for the Linear Interpolation: Since $\left|H^{\prime \prime}(f)\right|$ in (20) follows a Rayleigh distribution, the joint $\operatorname{pdf} p_{\xi, r}(\xi, r)$ of the channel and the linear interpolation follows the distribution given by (7). We now express the correlation coefficient $\rho_{r \xi, l i}$ between the channel and its linear interpolation error:

$$
\begin{aligned}
\rho_{r \xi, l i} & =\frac{\left|E\left\{H(f) e_{h}^{*}\right\}\right|}{\sigma_{h} \sigma_{\xi_{l i}}} \\
& =\frac{\left|\left(f_{p+\delta_{p}}-f\right)\left(f_{p}-f\right)\right| 2 \pi^{2} \sum_{l=1}^{L-1} \tau_{l}^{2} \sigma_{l}^{2}}{\sigma_{h} \sigma_{\xi_{l i}}} .
\end{aligned}
$$

Like in the NN case, we notice from (24) that $\rho_{r \xi, l i}$ depends on $f$. We directly derive the discrete expression of the correlation coefficient depending on $k$ and noted $\rho_{r \xi_{k}, l i}$ :

$$
\rho_{r \xi_{k}, l i}=\frac{\left|\frac{\left(\delta_{p}-k\right) k}{M^{2}}\right| 2 \pi^{2} \sum_{l=1}^{L-1} \beta_{l}^{2} \sigma_{l}^{2}}{\sigma_{r} \sigma_{\xi_{k, l i}}} .
$$




\section{Piecewise Cubic Interpolation}

1) Reminder: We now consider the piecewise cubic polynomial interpolation made with the Lagrange basis polynomials $\left\{\mathcal{L}_{p}\right\}$ defined by

$$
\left\{\begin{array}{l}
\mathcal{L}_{p}(f)=\frac{f-f_{p+\delta_{p}}}{f_{p}-f_{p+\delta_{p}}} \times \frac{f-f_{p+2 \delta_{p}}}{f_{p}-f_{p+2 \delta_{p}}} \times \frac{f-f_{p+3 \delta_{p}}}{f_{p}-f_{p+3 \delta_{p}}} \\
\mathcal{L}_{p+\delta_{p}}(f)=\frac{f-f_{p}}{f_{p+\delta_{p}}-f_{p}} \times \frac{f-f_{p+2 \delta_{p}}}{f_{p+\delta_{p}}-f_{p+2 \delta_{p}}} \times \frac{f-f_{p+3 \delta_{p}}}{f_{p+\delta_{p}}-f_{p+3 \delta_{p}}} \\
\mathcal{L}_{p+2 \delta_{p}}(f)=\frac{f-f_{p}}{f_{p+2 \delta_{p}}-f_{p}} \times \frac{f-f_{p+\delta_{p}}}{f_{p+2 \delta_{p}}-f_{p+\delta_{p}}} \times \frac{f-f_{p+3 \delta_{p}}}{f_{p+2 \delta_{p}}-f_{p+3 \delta_{p}}} \\
\mathcal{L}_{p+3 \delta_{p}}(f)=\frac{f-f_{p}}{f_{p+3 \delta_{p}}-f_{p}} \times \frac{f-f_{p+\delta_{p}}}{f_{p+3 \delta_{p}}-f_{p+\delta_{p}}} \times \frac{f-f_{p+2 \delta_{p}}}{f_{p+3 \delta_{p}}-f_{p+2 \delta_{p}}} .
\end{array}\right.
$$

The interpolated channel $\hat{H}(f)$ build thanks to the Lagrange basis (26) and defined on $\left[f_{p}, f_{p+3 \delta_{p}}\right]$ is then given by

$$
\hat{H}(f)=\sum_{n=0}^{3} H\left(f_{p}+n \delta_{p}\right) \mathcal{L}_{p+n \delta_{p}}(f) .
$$

The whole estimated channel is then obtained by performing (27) on each interval $\left[f_{0}, f_{3 \delta_{p}}\right],\left[f_{3 \delta_{p}}, f_{6 \delta_{p}}\right]$, $\ldots,\left[f_{M-1-3 \delta_{p}}, f_{M-1}\right]$ extracted from $\left[f_{0}, f_{M-1}\right]$.

2) Variance of the Errors for the Piecewise Cubic Interpolation: In the same way as the linear interpolation, it is known that, thanks to a Taylor's expansion of a function $g$ of class $\mathcal{C}^{4}$, the error of interpolation in the case of a Lagrange cubic interpolation is given by

$$
|g(x)-\hat{g}(x)|=\frac{1}{24}\left|\prod_{n=0}^{3}\left(x-x_{p+n \delta_{p}}\right)\right| \times\left|g^{(4)}(y)\right|,
$$

where $g^{(4)}$ is the fourth derivative of $g$ and $y$ is in $\left[x_{p}, x_{p+n \delta_{p}}\right]$. As we notice that the channel frequency response (2) is $\mathcal{C}^{4}$, we replace $g(x)$ by $H(f)$ in (28) to get the expression of the error:

$$
\xi_{c u}=\frac{1}{24}\left|\prod_{n=0}^{3}\left(f-f_{p+n \delta_{p}}\right)\right| \times\left|H^{(4)}(\alpha)\right| .
$$

From (29), we deduce that $\xi_{c u}$ follows a Rayleigh distribution and, by using the same development as from (19) to (22), we obtain the variance of the error $\sigma_{\xi_{c u}}^{2}$ in the continuous formalism:

$$
\sigma_{\xi, c u}^{2}=\frac{1}{24^{2}}\left|\prod_{n=0}^{3}\left(f-f_{p+n \delta_{p}}\right)\right|^{2} \times 2^{8} \pi^{8} \sum_{l=1}^{L-1} \sigma_{l}^{2} \tau_{l}^{8} .
$$

The discrete formalism slightly differs from the one described on Fig. 2 in the case of the piecewise cubic interpolation, since each interval of interpolation is composed of four pilots instead of two. Thus, let us now consider frequency positions $m=p+k$ in the discrete interval $\llbracket p, p+3 \delta_{p} \rrbracket$, as described on Fig. for $\delta_{p}=2$. 


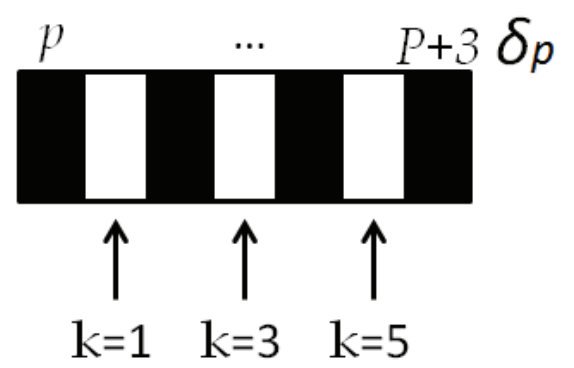

Fig. 3. Discrete representation of an interval for the piecewise cubic interpolation, with $\delta_{p}=2$.

The discrete expression of the error variance is written

$$
\sigma_{\xi_{k, c u}}^{2}=\frac{1}{24^{2}}\left|\frac{\prod_{n=0}^{3}\left(n \delta_{p}-k\right)}{M^{4}}\right|^{2} \times 2^{8} \pi^{8} \sum_{l=1}^{L-1} \sigma_{l}^{2} \beta_{l}^{8} .
$$

We observe on Fig. 3 that the interval $\llbracket p, p+3 \delta_{p} \rrbracket$ has a central symmetry, hence we deduce the general expression of the probability density of $\xi_{c u}$ as a sum of weighted Rayleigh distributions, by following the same reasoning leading to (6):

$$
p_{\xi_{c u}}\left(\xi_{c u}\right)= \begin{cases}\frac{1}{3\left(\delta_{p}-1\right)} \frac{\xi_{c u}}{\sigma_{\xi_{\delta_{p}}+\delta_{p} / 2, c u}} e^{-\frac{\xi_{c u}^{2}}{2 \sigma_{\xi_{p}}^{2}+\delta_{p} / 2, c u}}+\frac{2}{3\left(\delta_{p}-1\right)} \sum_{\substack{k=1 \\ k \neq \delta_{p}}}^{\left(\delta_{p}+\delta_{p} / 2\right)-1} \frac{\xi_{c u}}{\sigma_{\xi_{k, c u}}^{2}} e^{-\frac{\xi_{c u}^{2}}{2 \sigma_{\xi}, c u}} & , \text { if } \delta_{p} \text { is even } \\ \frac{2}{3\left(\delta_{p}-1\right)} \sum_{\substack{k=1 \\ k \neq \delta_{p}}}^{\delta_{p}+\left(\delta_{p}-1\right) / 2} \frac{\xi_{c u}}{\sigma_{\xi_{k, c u}}^{2}} e^{-\frac{\xi_{c u}^{2}}{2 \sigma_{\xi_{k, c u}}^{2}}} & \text { if } \delta_{p} \text { is odd }\end{cases}
$$

For each value $k$, the weight $\frac{2}{3\left(\delta_{p}-1\right)}$ is the ratio between the number of symmetrical subcarriers on the number of interpolated subcarriers in $\llbracket p, p+3 \delta_{p} \rrbracket$.

3) Correlation coefficient $\rho_{r \xi}$ for the Piecewise Cubic Interpolation: Since $\left|H^{(4)}(f)\right|$ follows a Rayleigh distribution, and by analogy with (25), we directly deduce the expression of the correlation coefficient in the case of the cubic interpolation:

$$
\rho_{r \xi_{k, c u}}=\frac{\left|\prod_{n=0}^{3}\left(n \delta_{p}-k\right)\right| 2^{4} \pi^{4} \sum_{l=1}^{L-1} \sigma_{l}^{2} \beta_{l}^{4}}{24 M^{4} \sigma_{r} \sigma_{\xi_{c u, k}}} .
$$

Finally, the generalized expression of the conditional pdf $p_{\xi_{k, c u} \mid r}\left(\xi_{k, c u} \mid r\right)$ for the cubic interpolation is the one of (8) in which the weights are those of (32).

In this section, it has been shown that a general expression of the interpolation errors statistics can be analytically derived. We then applied the analysis to three interpolation methods. By extrapolation, we deduce that it is possible to express the statistical properties of the interpolation errors as soon as the interpolation has an analytical expression of its error. In the following, we use these results to predict the mean square error of the LS estimator when performed with an interpolation. 


\section{Mean Square ERror of LS Estimation with Interpolation}

In [24], it is assumed that the mean square error of the estimations performed by LS with interpolations cannot be analytically expressed. Thus, the different methods are compared by means of simulations. In this section, thanks to the previous results concerning the statistics of the interpolations errors, we show that a theoretical expression of the MSE can be derived. To this end, since the interpolation of the channel is independent from the one of the noise, we now study the statistics of $\left(\frac{W_{m}}{C_{m}}\right)^{i n t}$ (3) separately. It is assumed that $\left|C_{m}\right|=1$, so $\left(\frac{W_{m}}{C_{m}}\right)^{i n t}$ has the same statistics as $W_{m}$, and we simply note $\hat{W}_{m}^{i n t}=\left(\frac{W_{m}}{C_{m}}\right)^{i n t}$ in the following.

\section{A. Statistics of the Interpolated Noise}

1) NN-Interpolated Noise: Whatever the values of $\delta_{p}$ and $k$, and for a fixed value $p$, the interpolated noise sample $\hat{W}_{m}^{i n t}$ such as with $m=p+k$, is expressed by

$$
\hat{W}_{m}^{i n t}=W_{p} / C_{p}
$$

From (34), we simply deduce that the $\mathrm{NN}$-interpolated noise has exactly the same statistics as the initial noise $\hat{W}_{m}$, i.e. $\hat{W}_{m}^{i n t} \sim \mathcal{N}\left(0, \sigma^{2}\right)$.

2) Linear-Interpolated Noise: Using the linear interpolation (16) in the discrete formalism and without taking the channel into account, the expression of $\hat{W}_{m}^{i n t}$ with $m=p+k$ is derived as:

$$
\hat{W}_{m}^{i n t}=W_{p} / C_{p}+k \frac{W_{p+1} / C_{p+\delta_{p}}-W_{p} / C_{p}}{\delta_{p}} .
$$

As whatever $p=0, \delta_{p}, . ., M-1$, we have $E\left\{W_{p}\right\}=0$, we directly deduce that $E\left\{\hat{W}_{m}^{i n t}\right\}=0$. Since $k$ appears in (35), the variance of the linear interpolated noise, noted $\sigma_{l i, k}^{2}$, is a function of $k$ such as

$$
\begin{aligned}
\sigma_{l i, k}^{2} & =E\left\{\left|\hat{W}_{m}^{i n t}\right|^{2}\right\} \\
& =E\left\{\left|\frac{W_{p} / C_{p}\left(\delta_{p}-k\right)+k W_{p+1} / C_{p+\delta_{p}}}{\delta_{p}}\right|^{2}\right\} .
\end{aligned}
$$

Reminding that the noise samples have the same variance and are uncorrelated, we get

$$
\sigma_{l i, k}^{2}=\frac{\left(\delta_{p}-k\right)^{2}+k^{2}}{\delta_{p}^{2}} \sigma^{2} .
$$

The total variance of $\hat{W}_{m}^{i n t}$, noted $\sigma_{l i}^{2}$ is the weighted sum of $\sigma_{l i, k}^{2}$ :

$$
\sigma_{l i}^{2}=\left\{\begin{array}{ll}
\frac{1}{\delta_{p}-1} \sigma_{l i, \delta_{p} / 2}^{2}+\frac{2}{\delta_{p}-1} \sum_{k=1}^{\delta_{p} / 2-1} \sigma_{l i, k}^{2}, & \text { if } \delta_{p} \text { is even } \\
\frac{2}{\delta_{p}-1} \sum_{k=1}^{\left(\delta_{p}-1\right) / 2} \sigma_{l i, k}^{2}, & \text { if } \delta_{p} \text { is odd }
\end{array} .\right.
$$


3) Piecewise Cubic-Interpolated Noise: By using the Lagrange cubic interpolation (27) applied to the noise samples, we obtain the variance of the interpolated noise $\sigma_{l i, k}^{2}$ as a function of $k$ :

$$
\begin{aligned}
\sigma_{c u, k}^{2} & =E\left\{\left|\hat{W}_{m}^{i n t}\right|^{2}\right\} \\
& =E\left\{\left|\sum_{n=0}^{3} W\left(p+n \delta_{p}\right) \mathcal{L}_{p+n \delta_{p}}(p+k)\right|^{2}\right\} \\
& =\sum_{n=0}^{3} \mathcal{L}_{p+n \delta_{p}}(p+k)^{2} \sigma^{2} .
\end{aligned}
$$

The total variance of $\hat{W}_{m}^{i n t}$, noted $\sigma_{c u}^{2}$ is the weighted sum of $\sigma_{c u, k}^{2}$ :

$$
\sigma_{c u}^{2}=\left\{\begin{array}{ll}
\frac{1}{3\left(\delta_{p}-1\right)} \sigma_{c u, \delta_{p}+\delta_{p} / 2}^{2}+\frac{2}{3\left(\delta_{p}-1\right)} \sum_{\substack{k=1 \\
k \neq \delta_{p}}}^{\left(\delta_{p}+\delta_{p} / 2\right)-1} \sigma_{c u, k}^{2}, & \text { if } \delta_{p} \text { is even } \\
\frac{2}{3\left(\delta_{p}-1\right)} \sum_{\substack{k=1 \\
k \neq \delta_{p}}}^{\left(\delta_{p}+\delta_{p} / 2\right)-1} \sigma_{c u, k}^{2}, & \text { if } \delta_{p} \text { is odd }
\end{array} .\right.
$$

In this first part, the interpolation errors and the interpolated noise have been statistically characterized. This can now be used to express the MSE of the LS with interpolations estimation.

\section{B. Analytical Expression of the MSE of the LS Estimations Performed with Interpolation}

The mean square error of a given interpolation is noted $M S E_{\text {int }}$ and is written

$$
M S E_{\text {int }}=\frac{1}{M} E\left\{\|\hat{\mathbf{H}}-\mathbf{H}\|^{2}\right\}
$$

Each OFDM symbol being composed of pilot tones and data carriers, we split the development into two parts. On each pilot tone, the MSE has already been expressed in [21], [24]. In that case, the MSE, denoted by $M S E_{p}$, is given by

$$
M S E_{p}=\frac{\sigma^{2}}{\mathcal{P}}
$$

with $\mathcal{P}$ the pilots power. Although in this article, it is assumed that $\mathcal{P}=1$, we keep the notation $\mathcal{P}$ in the further developments to provide a general expression of the MSE. On the data carriers $m \neq p$, the MSE is noted $M S E_{d}$, and from (3), we obtain

$$
M S E_{d}=E\left\{\left|\hat{H}_{m}^{i n t}+\left(\frac{W_{m}}{C_{m}}\right)^{i n t}-H_{m}\right|^{2}\right\},
$$

and as the noise and the channel are uncorrelated, it yields 


$$
\begin{aligned}
M S E_{d} & =E\left\{\left|\hat{H}_{m}^{i n t}-H_{m}\right|^{2}\right\}+E\left\{\left|\left(\frac{W_{m}}{C_{m}}\right)^{i n t}\right|^{2}\right\} \\
& =\sigma_{\xi}^{2}+\frac{\sigma_{i n t}^{2}}{\mathcal{P}},
\end{aligned}
$$

where $\sigma_{i n t}^{2}$ is equal to $\sigma^{2}, \sigma_{l i}^{2}(38)$ and $\sigma_{c u}^{2}(40)$ for the $\mathrm{NN}$, the linear and the piecewise cubic interpolations, respectively. Finally, the MSE is obtained by inserting (44) and (42) in (41):

$$
M S E_{i n t}=\frac{N_{p}}{M} \frac{\sigma^{2}}{\mathcal{P}}+\frac{M-N_{p}}{M}\left(\sigma_{\xi}^{2}+\frac{\sigma_{i n t}^{2}}{\mathcal{P}}\right),
$$

where $N_{p}$ is the number of pilot tones in an OFDM symbol. When performed on a preamble, we know from [21] that the MSE of LS tends to zero for high SNR values. We notice in (45) that $M S E_{i n t}$ tends to $\frac{M-N_{p}}{M} \sigma_{\xi}^{2}$ for high SNR values. This is the result of the residual error due to the interpolations.

Fig. 4 displays the pdfs of the interpolated noise for the three considered methods, assuming a frequency gap $\delta_{p}=2$. The original noise has a variance $\sigma^{2}=1$, as depicted by its pdf. As expected, we observe that the $\mathrm{NN}$-interpolated noise has the same variance as the original one, whereas the linear and cubic interpolations reduce the noise variance. We should also notice that the linear-interpolated noise has a lower variance than the cubic. Consequently, we deduce that the former interpolator makes the LS estimation less sensitive to the noise than the latter one. This observation will be confirmed in terms of performance in Section VI.

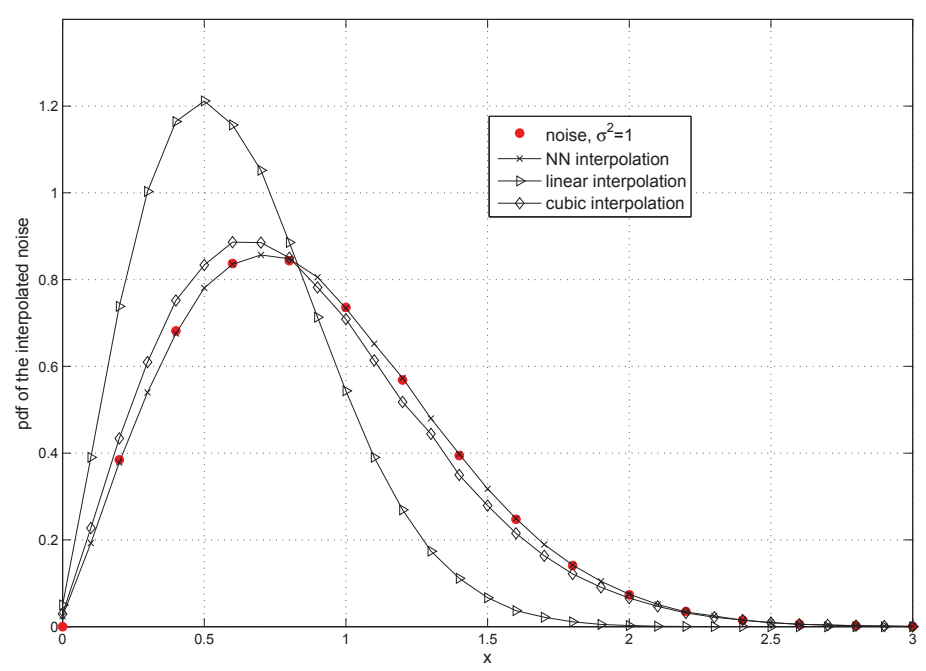

Fig. 4. Pdfs of the interpolated noise, for three interpolations methods, and for $\delta_{p}=2$, and $\sigma^{2}=1$. 


\section{Bit ERror RATE FLOOR OF LS Estimation With INTERPolation}

\section{A. Geometrical Considerations}

1) Reminder: In this section, the error floor reached by the BER curves for high SNR values is analytically evaluated when interpolation errors occur. In order to only consider the residual errors due to the interpolations, it is assumed high SNR values, so the noise is then neglected in this section. Since we focus on the effects of the interpolations errors on the BER, the geometry of the constellation has to be taken into account. In the following, a BPSK $(C \in\{-1,+1\})$ and a 4-QAM $\left(C \in\left\{{ }_{-}^{+} d_{-}^{+} j d\right\}\right)$ mapped with a Gray encoding are considered, with $C$ a transmitted symbol and $d$ a normalization coefficient.

At the receiver side, the channel is estimated by means of LS with interpolations. Then, the received symbol is estimated by a simple zero forcing equalizer. As the noise is supposed to be null, the estimated symbol $\hat{C}$ is written as follows

$$
\hat{C}=\frac{H(f)}{H(f)+e_{h}} C .
$$

For a BPSK, the decision domains are exactly symmetric for $C={ }_{-}^{+}$. Let us then set $C=+1$ in the following developments. The probability of detection error, noted $P_{e}^{B P S K}$ is then given by

$$
P_{e}^{B P S K}=\operatorname{Pr}(\mathfrak{R e}\{\hat{C}\}<0 \mid C=1) .
$$

In the same way for a 4-QAM, and as mentioned in [14], the decision domains are exactly symmetric with regard to the Q and I axes for the most significant bit (MSB) and for the least significant bit (LSB), respectively. Thus, let us set $C=+d+j d$ in the following developments. In that case, the probability of error of detection, noted $P_{e}^{4 Q A M}$ is then given by

$$
P_{e}^{4 Q A M}=\operatorname{Pr}(\mathfrak{R e}\{\hat{C}\}<0 \mid C=d+j d) .
$$

For a given value $|H(f)|=r$, we denote the complex expressions of the channel $H(f)=r e^{j \Theta_{H}}$ and the error $e_{h}=\xi e^{j \Theta_{\xi}}$, and we define $\Theta=\Theta_{H}-\Theta_{\xi}$. Let us now derive the analytical expression of (47) and (48).

2) BPSK: After some straightforward developments, the inequality in (47) yields

$$
\begin{aligned}
& \mathfrak{R e}\{\hat{C}\}<0 \text { with } C=1 \\
& \Leftrightarrow \mathfrak{R e}\left\{\frac{H(f)}{H(f)+e_{h}} \times 1\right\}<0 \\
& \Leftrightarrow \frac{r^{2}+r \xi \cos (\Theta)}{\left|H(f)+e_{h}\right|^{2}}<0 .
\end{aligned}
$$


Since $\left|H(f)+e_{h}\right|^{2}>0$, we limit the study to the numerator of (49). It can be seen that if $\xi \leq r$, then $\forall \Theta \in[0,2 \pi]$ the inequality cannot be valid because $-1 \leq \cos (\Theta) \leq 1$. Thus, $P_{e}^{B P S K}=0$ as long as $\xi \leq r$. For $\xi \geq r$, the inequality (49) is valid for

$$
\Theta \in\left[-\arccos \left(-\frac{r}{\xi}\right), \arccos \left(-\frac{r}{\xi}\right)\right] .
$$

As $\Theta$ is defined in $[0,2 \pi]$, we then express $P_{e}^{B P S K}$ for $\xi \geq r$ as a function of $r$ and $\xi$

$$
\begin{aligned}
P_{e}^{B P S K} & =\frac{\arccos \left(-\frac{r}{\xi}\right)-\left(-\arccos \left(-\frac{r}{\xi}\right)\right)}{2 \pi} \\
& =1-\frac{\arccos \left(-\frac{r}{\xi}\right)}{\pi},
\end{aligned}
$$

and then, finally, $P_{e}^{B P S K}$ is expressed by

$$
P_{e}^{B P S K}=\left\{\begin{array}{ll}
0 & \text { if } \xi \leq r \\
1-\frac{\arccos \left(-\frac{r}{\xi}\right)}{\pi} & \text { if } \xi \geq r
\end{array} .\right.
$$

As $\frac{\arccos (1)}{\pi}=1$, then $P_{e}^{B P S K}$ is a continuous function on $\mathbb{R}^{+}$. From the conditional pdf $p_{\xi \mid r}(\xi \mid r)$, we extract the conditional pdf of errors of estimation causing a wrong decision on the bits for a BPSK, that we denote $\Phi_{\xi \mid r, B P S K}(\xi \mid r, \mathfrak{R e}\{\hat{C}\} \neq \mathfrak{R e}\{C\})$ :

$$
\Phi_{\xi \mid r, B P S K}(\xi \mid r, \mathfrak{R e}\{\hat{C}\} \neq \mathfrak{R e}\{C\})=P_{e}^{B P S K} p_{\xi \mid r}(\xi \mid r) .
$$

Fig. 5 (a) displays the Rayleigh density $p_{\xi \mid r}(\xi \mid r)$ for $r=1, \sigma_{\xi}^{2}=1, \rho_{r \xi}=0$ (i.e. $p_{\xi}(\xi)$ ) and the extracted function $\Phi_{\xi \mid r, B P S K}(\xi \mid r, \mathfrak{R e}\{\hat{C}\} \neq \mathfrak{R e}\{C\})$, drawn by simulations and by means of (53). The curves are obtained after the transmission of $10^{6}$ bits.

It is verified that $\Phi_{\xi \mid r, B P S K}(\xi \mid r, \mathfrak{R e}\{\hat{C}\} \neq \mathfrak{R e}\{C\})=0$ for $\xi \leq r$, with $r=1$ in the simulation. Furthermore, the theoretical curve and the one drawn by simulations exactly match, which validates the previous developments.

3) 4-QAM Constellation: After some developments, the inequality $\mathfrak{R e}\{\hat{C}\}<0$ in (48) yields

$$
\begin{aligned}
& \mathfrak{R e}\{\hat{C}\}<0 \\
& \Leftrightarrow \mathfrak{R e}\left\{H(f)\left(H(f)+e_{h}\right)^{*}(1+j)\right\}<0 \\
& \Leftrightarrow \mathfrak{R e}\left\{\left(r^{2}+r \xi e^{j\left(\Theta_{H}-\Theta_{\xi}\right)}\right)(1+j)\right\}<0 \\
& \Leftrightarrow r+\xi(\cos (\Theta)-\sin (\Theta))<0
\end{aligned}
$$

If we set $t=\tan \left(\frac{\Theta}{2}\right)$, we then get $\cos (\Theta)=\frac{1-t^{2}}{1+t^{2}}$ and $\sin (\Theta)=\frac{2 t}{1+t^{2}}$, and (54) yields 


$$
\frac{r\left(1+t^{2}\right)+\xi\left(1-t^{2}-2 t\right)}{1+t^{2}}<0 .
$$

Since $1+t^{2}>0$ whatever the value of $t$, we study the sign of the numerator in (55), and we denote $Q$ the polynomial:

$$
Q(t)=t^{2}(r-\xi)-2 \xi t+r+\xi
$$

The solving of the inequality (55) is made by studying the convexity or concavity of $Q$ and by looking for its roots. We finally obtain:

$$
P_{e}^{4 Q A M}=\left\{\begin{array}{l}
0, \text { if } 0 \leq \xi \leq \frac{r}{\sqrt{2}} \\
\frac{\arctan \left(t_{2}^{+}\right)-\arctan \left(t_{2}^{-}\right)}{\pi}, \text { if } \frac{r}{\sqrt{2}} \leq \xi \leq r \\
1+\frac{\arctan \left(t_{3}^{-}\right)-\arctan \left(t_{3}^{+}\right)}{\pi}, \text { if } r \leq \xi
\end{array}\right.
$$

where $t_{2}^{ \pm}$and $t_{3}^{ \pm}$are expressed by

$$
\begin{aligned}
& t_{2}^{ \pm}=\frac{2 \xi_{-}^{+} \sqrt{\Delta_{p}}}{2(r-\xi)}, \text { and } \\
& t_{3}^{ \pm}=\frac{2 \xi_{+}^{-} \sqrt{\Delta_{p}}}{2(r-\xi)},
\end{aligned}
$$

with $\Delta_{p}=4 \xi^{2}-4\left(r^{2}-\xi^{2}\right)$ the discriminant of $Q$. It is straightforward to show that $P_{e}^{4 Q A M}$ is continuous on $\mathbb{R}^{+}$by means of a Taylor's expansion on $r=\xi$. From the conditional pdf $p_{\xi \mid r}(\xi \mid r)$, we extract the pdf of errors of estimation causing wrong decision on the bits for a 4-QAM, that we denote $\Phi_{\xi, 4 Q A M}(\xi \mid r, \mathfrak{R e}\{\hat{C}\} \neq \mathfrak{R e}\{C\})$. Since the I and Q components of the 4-QAM constellation are symmetric, we simply get

$$
\Phi_{\xi, 4 Q A M}(\xi \mid r, \mathfrak{R e}\{\hat{C}\} \neq \mathfrak{R e}\{C\})=P_{e}^{4 Q A M} p_{\xi \mid r}(\xi \mid r) .
$$

Fig. 5 (b) depicts the Rayleigh density $p_{\xi \mid r}(\xi \mid r)$ and the function $\Phi_{\xi \mid r, 4 Q A M}(\xi \mid r, \mathfrak{R e}\{\hat{C}\} \neq \mathfrak{R e}\{C\})$ drawn with the same parameters as previously. Once more, it is verified that the theoretical curve and the one drawn by simulation perfectly match.

\section{B. Analytical Expression of the BER Floor}

The bit error rate floor, noted $B E R^{\text {floor }}$, is the double integral of the conditional $\operatorname{pdf} \Phi_{\xi \mid r, \text { const }}(\xi \mid r, \mathfrak{R e}\{\hat{C}\} \neq$ $\mathfrak{R e}\{C\})$ multiplied by $p_{r}(r)$. Some simplifications lead to: 


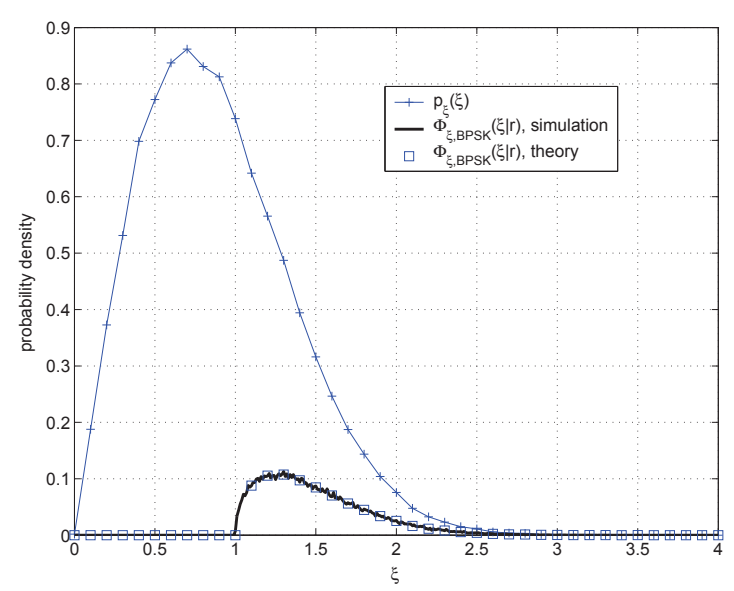

(a) Extracted conditional pdf $\Phi_{\xi \mid r, B P S K}$

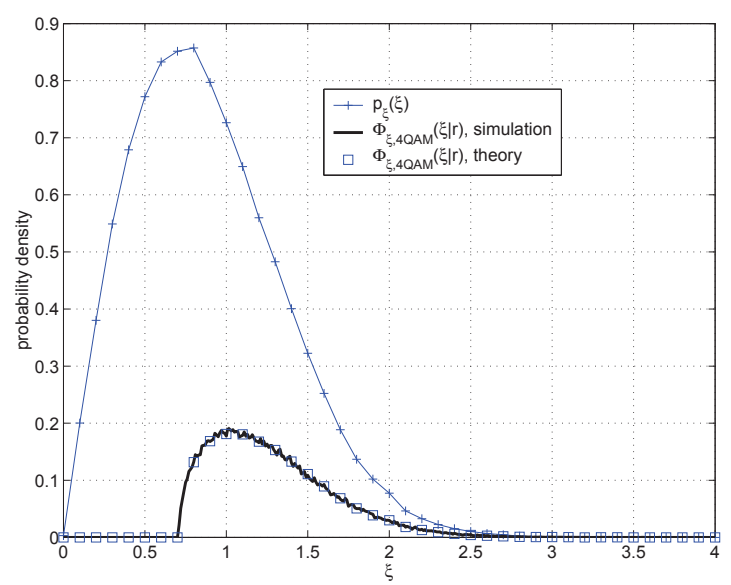

(b) Extracted conditional pdf $\Phi_{\xi \mid r, 4 Q A M}$

Fig. 5. Extracted conditional pdfs, (a) for a BPSK and (b) for a 4-QAM.

$$
\begin{aligned}
B E R^{\text {floor }} & =\int_{0}^{+\infty} p_{r}(r) \int_{0}^{+\infty} \Phi_{\xi \mid r, \text { const }}(\xi \mid r, \mathfrak{R e}\{\hat{C}\} \neq \mathfrak{R e}\{C\}) d \xi d r \\
& =\int_{0}^{+\infty} p_{r}(r) \int_{0}^{+\infty} P_{e}^{\text {const }} p_{\xi \mid r}(\xi \mid r) d \xi d r \\
& =\int_{0}^{+\infty} \int_{0}^{+\infty} P_{e}^{\text {const }} p_{r, \xi}(r, \xi) d \xi d r,
\end{aligned}
$$

where $P_{e}^{\text {const }}$ depends on the constellation (it is defined in (52) for the BPSK and in (57) for the 4-QAM).

\section{Simulations AND Discussion}

\section{A. Simulations Parameters}

In order to validate the previous developments by simulations, we do not limit us to a given standard, but we define two different general channels noted $\mathcal{H}_{(1)}$ and $\mathcal{H}_{(2)}$. Their parameters (the delay and variance of each non-null path) are summed up in Tables I and II. It can be seen that the number of paths, the maximum delay and the variance of the channels differ from $\mathcal{H}_{(1)}$ to $\mathcal{H}_{(2)}$.

\section{TABLE I}

Channel $\mathcal{H}_{(1)}$ Parameters.

\begin{tabular}{|l|l|l|l|l|}
\hline \multicolumn{5}{|c|}{ Channel $\mathcal{H}_{(1)}$} \\
\hline paths $l$ & 0 & 1 & 2 & 3 \\
\hline delays $\beta_{l}$ & 0 & 6 & 13 & 16 \\
\hline variance $\sigma_{l}^{2}$ & 1 & 0.5 & 0.4 & 0.2 \\
\hline
\end{tabular}


TABLE II

Channel $\mathcal{H}_{(2)}$ PARAMEters.

\begin{tabular}{|l|l|l|l|l|l|l|l|}
\hline \multicolumn{7}{|c|}{ Channel $\mathcal{H}_{(2)}$} \\
\hline paths $l$ & 0 & 1 & 2 & 3 & 4 & 5 & 6 \\
\hline delays $\beta_{l}$ & 0 & 4 & 9 & 14 & 16 & 18 & 21 \\
\hline variance $\sigma_{l}^{2}$ & 1 & 0.5 & 0.4 & 0.3 & 0.3 & 0.2 & 0.1 \\
\hline
\end{tabular}

\section{B. Mean Square Error}

Fig. 6 displays the MSE of the channel estimations performed with the NN, the linear (Figs. (a) and (b)) and the cubic (subfigures (c) and (d)) interpolations versus the SNR given in $\mathrm{dB}$. The theoretical curves given by (45) are compared to the ones drawn by simulation. The curves are obtained by means of 2400 simulations runs.

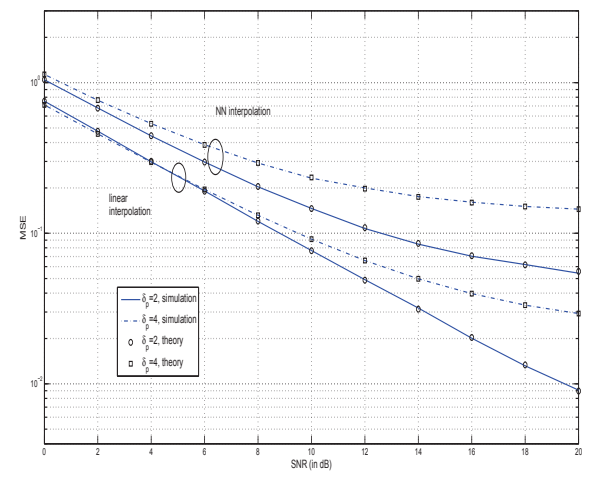

(a) Channel $\mathcal{H}_{(1)}$, NN and linear interpolations

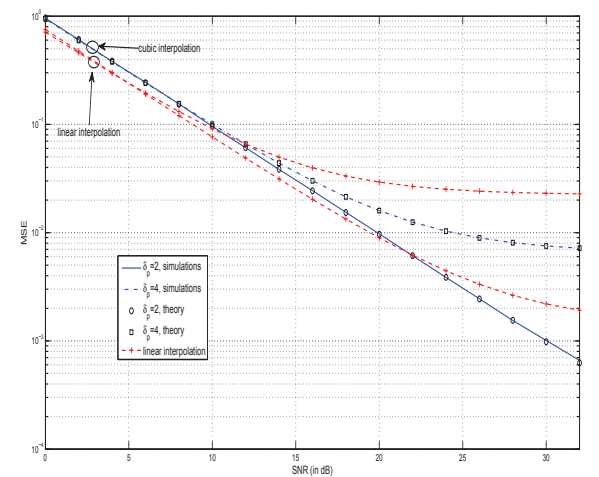

(c) Channel $\mathcal{H}_{(1)}$, linear and cubic interpolations

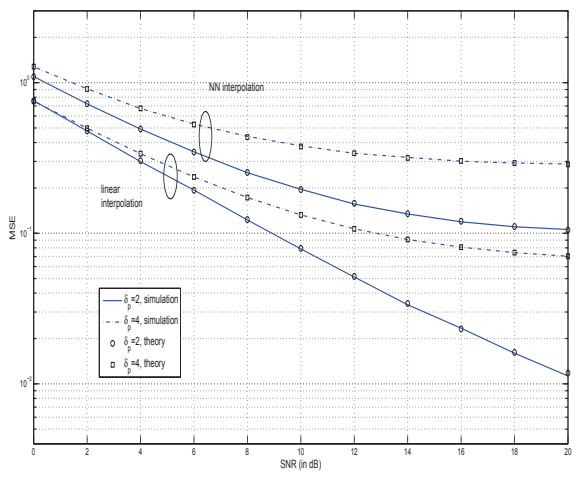

(b) Channel $\mathcal{H}_{(2)}, \mathrm{NN}$ and linear interpolations

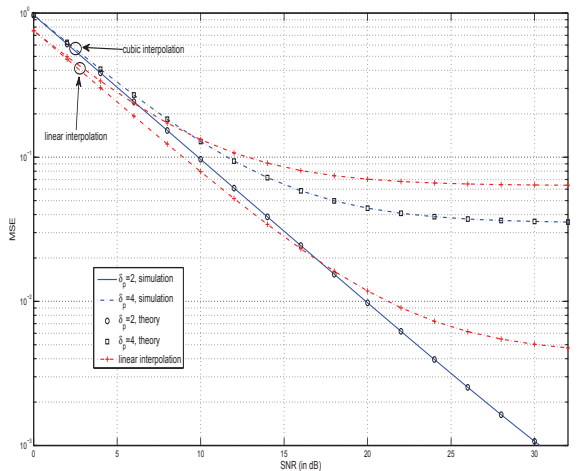

(d) Channel $\mathcal{H}_{(2)}$, linear and cubic interpolations

Fig. 6. MSE of the channel estimation performed with NN, linear and cubic interpolations, for $\delta_{p} \in\{2,4\}$, and for the channels $\mathcal{H}_{(1)}$ and $\mathcal{H}_{(2)}$. 
Firstly, we observe that the theoretical curves and the ones drawn by simulations perfectly match, which validates the theoretical results derived in this article. As expected, the MSE reaches an error floor that decreases when the polynomial order increases. Furthermore, these errors floors are higher for the channel $\mathcal{H}_{(2)}$ (Figs. (b) and (d)) than for the channel $\mathcal{H}_{(1)}$ (Figs. (a) and (c)). This is in accordance with the theory, since $\mathcal{H}_{(2)}$ is more frequency selective than $\mathcal{H}_{(1)}$.

Secondly, from Figs. (c) and (d), we observe that the linear interpolation has a lower MSE than the cubic one for low SNR values, whereas the cubic interpolator has a better performance than linear one for higher SNR values. It is explained by the aforementioned observation in regard to the variance of the interpolated noise made in Section IV. Indeed, at low SNR values, the noise effect prevails on the interpolation errors, and since the linear-interpolated noise has a lower variance than the cubic-interpolated noise, linear interpolation has a lower MSE. On the contrary, for higher SNR, interpolation errors effect prevails on the noise, and since the cubic interpolation is more accurate than the linear one, then the former has a lower MSE than the latter. These results will be discussed in Section VI-D.

\section{Bit Error Rate Low Bound}

Fig. 7 shows the simulated BER curves and the theoretical BER low bound values versus the SNR given in dB. Figs. 7 (a) - (b) display the BER curves versus the SNR for the NN interpolation, Figs. 7 (c) - (d) for the linear interpolation and Figs. 7 (e) - (f) for the cubic interpolation. In order to validate the proposed developments, the simulations are made for both channels $\mathcal{H}_{(1)}$ and $\mathcal{H}_{(2)}$, and for $\delta_{p} \in\{2,4\}$. The curves are obtained with $2 \times 10^{7}$ bits.

It can be seen on Fig. 7 that, whatever the chosen set of parameters, the simulated curves reach a BER floor matching the analytical values. The very slight differences can be explained by the fact that the integral (62) is computed in a discrete formalism. However, we clearly observe that the BER lower bound decreases when the degree of the polynomial interpolator increases. Furthermore, the performance is more degraded in $\mathcal{H}_{(2)}$ compared to $\mathcal{H}_{(1)}$, due to $\mathcal{H}_{(2)}$ is more frequency selective than $\mathcal{H}_{(1)}$. These results then allow to validate the theoretical developments proposed in this article.

\section{Discussion}

In addition to be a theoretical analysis of the LS estimator performance in a frequency selective Rayleigh channel by means of polynomial interpolations, we can deduce a practical implementation from the proposed method. Indeed, an adaptive receiver (in terms of estimation technique) can be implemented, according to the trade-off between performance and complexity. It is known that the $\mathrm{NN}$, the linear and the cubic interpolations require zero, two and four multiplications per interpolated value, respectively. Thus, for a given BER target, and given the frequency selectivity, the proposed 


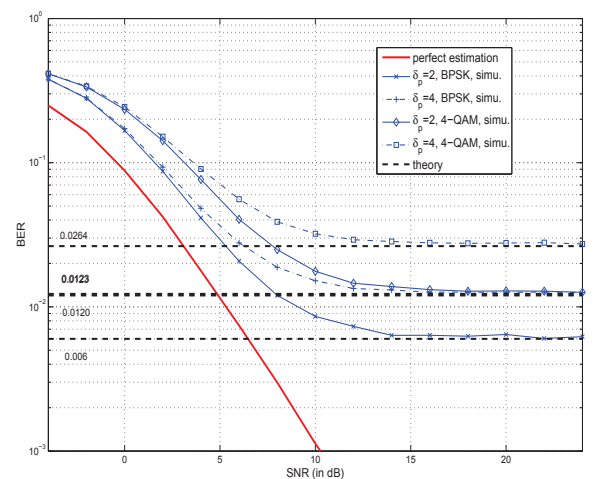

(a) $\mathrm{NN}$ interpolation, channel $\mathcal{H}_{(1)}$

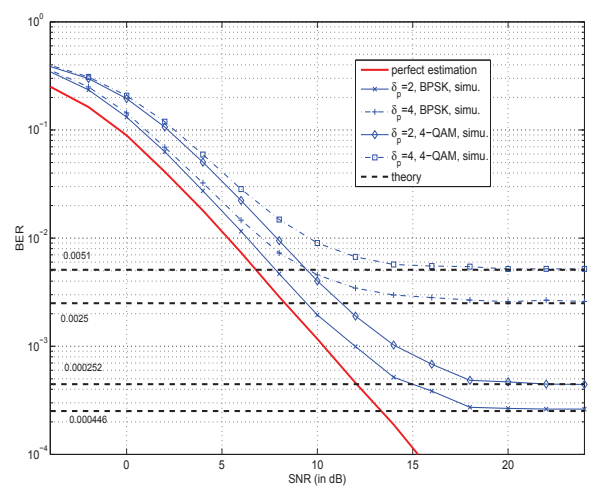

(c) Linear interpolation, channel $\mathcal{H}_{(1)}$

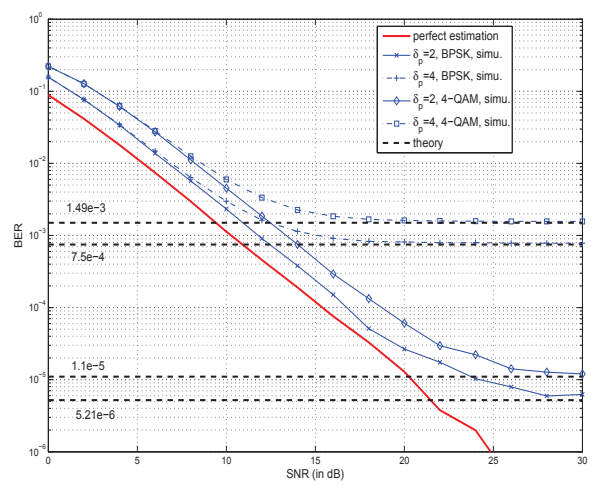

(e) Cubic interpolation, channel $\mathcal{H}_{(1)}$

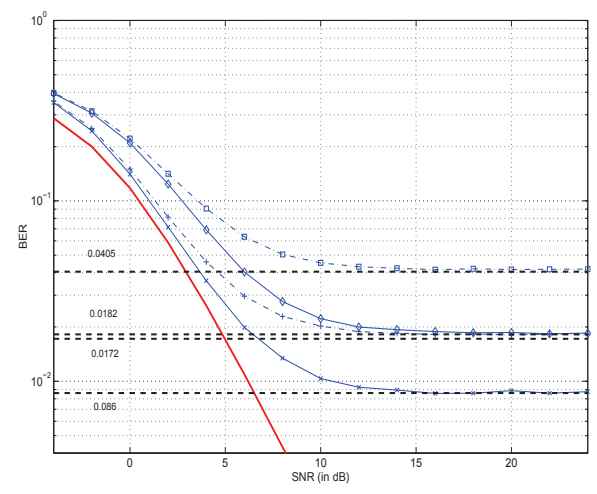

(b) NN interpolation, channel $\mathcal{H}_{(2)}$

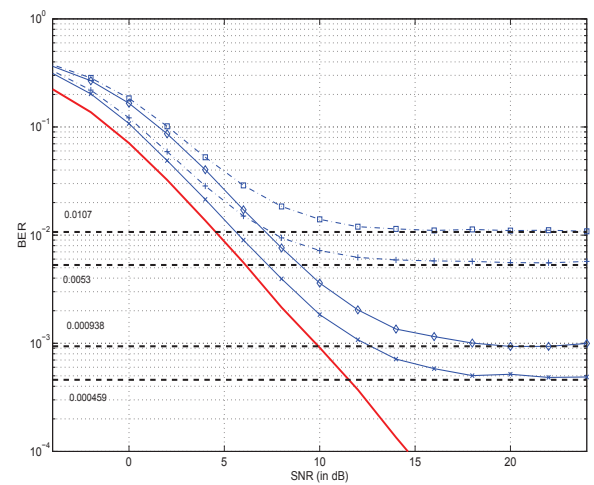

(d) Linear interpolation, channel $\mathcal{H}_{(2)}$

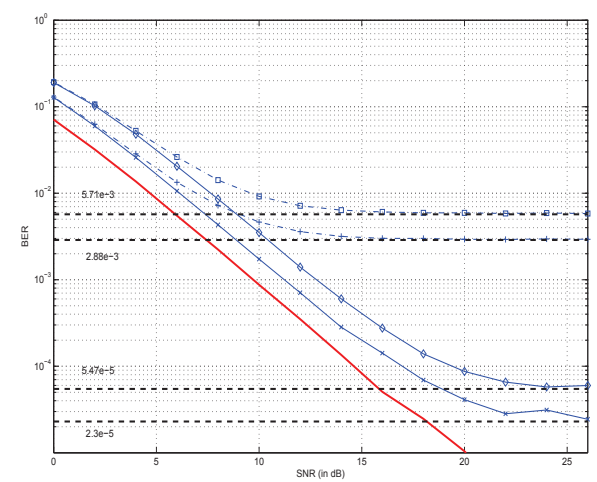

(f) Cubic interpolation, channel $\mathcal{H}_{(2)}$

Fig. 7. BER of the channel estimation performed with (a) - (b) NN interpolation, (c) - (d) linear interpolation and (e) - (f) cubic interpolation, for $\delta_{p} \in\{2,4\}$, and for the channels $\mathcal{H}_{(1)}$ and $\mathcal{H}_{(2)}$. 
method enables the receiver to choose the interpolation technique with minimum computation cost. Moreover, as it has been noticed for low SNR values, it may appear advantageous to perform the estimation by means of the linear interpolation instead of the cubic one, while it is not a priori intuitive. Besides, this study can be applied in other domains, for which interpolations of random functions are required, as in image processing for instance.

\section{CONCLUSION}

In this article, a theoretical study of the interpolation errors applied to the channel estimation has been presented. Firstly, a characterization of the errors statistics has been performed, and a geometrical analysis of the error effect on the constellation has been proposed. Then, an analytical expression of the MSE of the LS estimation performed with interpolation has been derived, just as the expression of the BER floor. For a given channel, we have shown that these values are functions of the frequency gap between the pilot tones $\delta_{p}$, the interpolation technique and the constellation size. The developments have been made for the polynomials of degree zero, one and three, and for the BPSK and the 4-QAM constellations as well. It has been shown that the theoretical results perfectly match the simulations. This analysis can then be used to a priori predict the performance of the system. It differs from the existing works of the literature, because the errors and the channel are correlated, and the MSE and BER can be exactly characterized. Beyond the theoretical performance analysis, we have proposed a possible practical implementation, in which the method is used to adapt the receiver according to the trade-off between the expected performance and the complexity of the interpolation technique.

\section{REFERENCES}

[1] S. Coleri, M. Ergen, A. Puri, and A. Bahai, “A study of channel estimation in OFDM systems," in Vehicular Technology Conference Fall, vol. 2, september 2002, pp. 894-898.

[2] X. Dong, W.-S. Lu, and A. Soong, "Linear Interpolation in Pilot Symbol Assisted Channel Estimation for OFDM," IEEE Trans. on wireless Communications, vol. 6, no. 5, pp. 1910 - 1920, May 2007.

[3] O. Edfors, M. Sandell, J.-J. van de Beek, S. K. Wilson, and P. O. Börjesson, "OFDM Channel Estimation by Singular Value Decomposition,” IEEE Trans. on Communications, vol. 46, no. 7, pp. 931 - 939, July 1998.

[4] M. Biguesh and A. B. Gershman, "Training-Based MIMO Channel Estimation: A Study of Estimator Tradeoffs and Optimal Training Signals," IEEE Transactions on Signal Processing, vol. 54, no. 3, pp. 884-893, March 2006.

[5] ETSI, "Digital Radio Mondiale (DRM);System Specification,” ETSI, Tech. Rep. ETSI ES 201980 V 3.1.1, August 2009.

[6] _ - "Digital video broadcasting (DVB) framing structure, channel coding and modulation for digital terrestrial television,” ETSI, Tech. Rep. ETSI EN 300744 V1.5.1, 2004.

[7] M.-S. Alouini and A. J. Goldsmith, "A Unified Approach for Calculating Error Rates of Linearly Modulated Signals over Generalized Fading Channels," IEEE Trans. on Communications, vol. 47, no. 9, pp. 1324 - 1334, September 1999.

[8] P. K. Vitthaladevuni and M. S. Alouini, “A closed-form expression for the exact BER of generalized PAM and QAM constellations," IEEE Trans. on Communications, vol. 52, no. 5, pp. 698 - 700, May 2004. 
[9] — - "A Recursive Algorithm for the Exact BER Computation of Generalized Hierarchical QAM Constellations," IEEE Transactions on Information Theory, vol. 59, no. 1, pp. 297 - 307, January 2003.

[10] X. Tang, M.-S. Alouini, and A. J. Goldsmith, "Effect of Channel Estimation Error on M-QAM BER Performance in Rayleigh Fading,” IEEE Trans. on Communications, vol. 47, no. 12, pp. 1856 - 1864, December 1999.

[11] L. Cao and N. C. Beaulieu, "Exact Error-Rate Analysis of Diversity 16-QAM With Channel Estimation Error," IEEE Trans. on Communications, vol. 52, no. 6, pp. 1019 - 1029, June 2004.

[12] A. Z. M. T. Islam and I. Misra, "Performance of Wireless OFDM System with LS-Interpolation-based Channel Estimation in Multi-path Fading Channel," International Journal on Computational Sciences \& Applications, vol. 2, no. 5, October 2012.

[13] M. Krondorf, T.-J. Liang, and G. Fettweis, "Symbol Error Rate of OFDM Systems with Carrier Frequency Offset and Channel Estimation Error in Frequency Selective Fading Channels," in ICC'07, Glasgow, 2007, pp. 5132 - 5136.

[14] C.-H. Yih, "Effects of Channel Estimation Error in the Presence of CFO on OFDM BER in Frequency-Selective Rayleigh Fading Channels," Journal of Communications, vol. 3, no. 3, pp. 10 - 18, July 2008.

[15] S. K. Wilson and J. M. Cioffi, "Probability Density Functions for Analyzing Multi-Amplitude Constellations in Rayleigh and Ricean Channels," IEEE Trans. on Communications, vol. 47, no. 3, pp. 380 - 386, March 1999.

[16] S. M. Kay, Fundamentals of Statistical Signal Processing : Estimation Theory. Prentice Hall, 2003.

[17] V. Savaux, Y. Louët, M. Djoko-Kouam, and A. Skrzypczak, "Minimum mean-square-error expression of LMMSE channel estimation in SISO OFDM systems," IET Electronics Letters, vol. 49, no. 18, pp. 1152 - 1154, August 2013.

[18] M.-H. Hsieh and C.-H. Wei, "Channel Estimation for OFDM Systems Based on Comb-Type Pilot Arrangement in Frequency Selective Fading Channels," IEEE Transations on Consumer Electronics, vol. 44, no. 1, pp. 217 - 225 , February 1998.

[19] M. K. Ozdemir and H. Arslan, "Channel Estimation for Wireless OFDM Systems," IEEE Communications Surveys \& Tutorials, vol. 9, no. 2, pp. 18 - 48, 2nd quarter 2007.

[20] P. Bello, "Characterization of randomly time-variant linear channels," IEEE Trans. on Communications Systems, vol. 11, no. 4, pp. 360-393, December 1963.

[21] M. Biguesh and A. B. Gershman, "Downlink Channel Estimation in Cellular Systems with Antenna Arrays at Base Stations Using Channel Probing with Feedback," EURASIP Journal on Applied Signal Processing, vol. 9, pp. 13301339, September 2004.

[22] Y. A. Chau and K. Y.-T. Huang, "On the Second-Order Statistics of Correlated Cascaded Rayleigh Fading Channels," International Journal of Antennas and Propagation, vol. 2012, p. 6, June 2012.

[23] M. K. Simon, Probability Distributions Involving Gaussian Random Variables: A Handbook For Engineers and Scientists. Springer, 2006.

[24] V. Savaux, Y. Louët, M. Djoko-Kouam, and A. Skrzypczak, "Artificial channel aided LMMSE estimation for timefrequency selective channels in OFDM context," Signal Processing, Elsevier, vol. 93, no. 9, pp. 2369 - 2380, September 2013. 